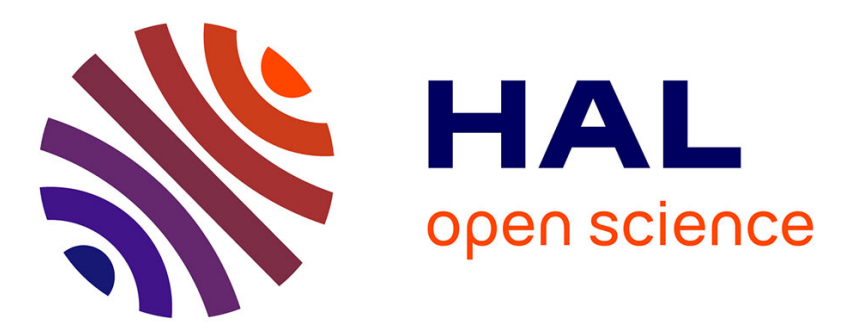

\title{
Segmentation of temporal mesh sequences into rigidly moving components
}

Romain Arcila, Cédric Cagniart, Franck Hétroy, Edmond Boyer, Florent

Dupont

\section{- To cite this version:}

Romain Arcila, Cédric Cagniart, Franck Hétroy, Edmond Boyer, Florent Dupont. Segmentation of temporal mesh sequences into rigidly moving components. Graphical Models, 2013, 75 (1), pp.10-22. 10.1016/j.gmod.2012.10.004 . hal-00749302

\section{HAL Id: hal-00749302 \\ https://hal.inria.fr/hal-00749302}

Submitted on 20 May 2016

HAL is a multi-disciplinary open access archive for the deposit and dissemination of scientific research documents, whether they are published or not. The documents may come from teaching and research institutions in France or abroad, or from public or private research centers.
L'archive ouverte pluridisciplinaire HAL, est destinée au dépôt et à la diffusion de documents scientifiques de niveau recherche, publiés ou non, émanant des établissements d'enseignement et de recherche français ou étrangers, des laboratoires publics ou privés. 


\title{
Segmentation of temporal mesh sequences into rigidly moving components
}

\author{
Romain Arcila ${ }^{\mathrm{a}, \mathrm{b}}$, Cédric Cagniart ${ }^{\mathrm{c}, \mathrm{a}}$, Franck Hétroy $^{\mathrm{a}, *}$, Edmond Boyer $^{\mathrm{a}}$, Florent Dupont ${ }^{\mathrm{b}}$ \\ ${ }^{a}$ Laboratoire Jean Kuntzmann, Inria $\mathcal{E}$ Grenoble University, France \\ ${ }^{b}$ LIRIS, CNRS $\mathcal{E}$ Université de Lyon, France \\ ${ }^{c}$ Computer Aided Medical Procedures $\mathcal{F}$ Augmented Reality (CAMPAR), Technische Universität München, Germany
}

\begin{abstract}
In this paper is considered the segmentation of meshes into rigid components given temporal sequences of deforming meshes. We propose a fully automatic approach that identifies model parts that consistently move rigidly over time. This approach can handle meshes independently reconstructed at each time instant. It allows therefore for sequences of meshes with varying connectivities as well as varying topology. It incrementally adapts, merges and splits segments along a sequence based on the coherence of motion information within each segment. In order to provide tools for the evaluation of the approach, we also introduce new criteria to quantify a mesh segmentation. Results on both synthetic and real data as well as comparisons are provided in the paper.
\end{abstract}

Keywords: mesh sequence, segmentation, topology, mesh matching, rigid part

\section{Introduction}

Temporal sequences of deforming meshes, also called mesh animations [1, 43], are widely used to represent 3D shapes evolving through time. They can be created from a single static mesh, which is deformed using standard animation techniques such as skeletal subspace deformation [25] or cloth simulation methods [15]. They can also be generated from multiple video cameras [38, 43]. In this case, meshes are usually independently estimated at each frame using 2D visual cues such as silhouettes or photometric information.

These deforming mesh sequences can be edited [21, 8], compressed [24], or used for deformation transfer [39, 23]. When the shape represents an articulated body, such as a human or animal character, identifying its rigid, or almost rigid, parts offers useful understanding for most of these applications. To recover the shape kinematic structure, an animation skeleton can be extracted from the deforming mesh sequence [1]. Another strategy is to segment the meshes into components that move rigidly over the sequence $[22,19,44,29]$. In both cases, motion information is required in order to cluster mesh elements into regions with rigid motions. Most existing approaches assume that surface registration is available for that purpose and consider as the input a single mesh that deforms over time. In contrast, we do not make any assumptions on the input mesh sequences and we propose to match meshes and recover their rigid parts simultaneously. Consequently, our method applies to any kind of deforming mesh sequence including inconsistent mesh sequences such as provided by multi-camera systems.

\footnotetext{
${ }^{*}$ Corresponding author.

Email addresses: Franck.Hetroy@grenoble-inp.fr (Franck Hétroy), Edmond.Boyer@inria.fr (Edmond Boyer),

Florent.Dupont@liris.cnrs.fr (Florent Dupont)
}

\subsection{Classification of mesh sequences}

In order to distinguish between mesh sequences with or without temporal coherence, i.e. with or without a one-to-one correspondence between vertices of successive meshes, we first introduce the following definitions.

Definition 1.1 (Temporally coherent mesh sequence (TCMS), temporally incoherent mesh sequence (TIMS)). Let $M S=$ $\left\{M^{i}=\left(V^{i}, E^{i}, F^{i}\right), i=1 \ldots f\right\}$ be a mesh sequence: $V^{i}$ is the set of vertices of the $i^{\text {th }}$ mesh $M^{i}$ of the sequence, $E^{i}$ its set of edges and $F^{i}$ its set of faces. If the connectivity is constant over the whole sequence, that is to say if there is an isomorphism between any $E^{i}$ and $E^{j}, 1 \leq i, j \leq f$, then $M S$ is called $a$ temporally coherent mesh sequence (TCMS). Otherwise, $M S$ is called $a$ temporally incoherent mesh sequence (TIMS).

Note that the definition of TCMS not only implies that the number of vertices remains constant through time, but also that there is a one-to-one correspondence between faces of any two meshes. As a consequence, topological changes (genus and number of connected components) are not possible in a TCMS.

Figure 1 shows an example of a TCMS and an example of a TIMS.

\subsection{Classification of mesh sequence segmentations}

In contrast to single mesh segmentation that consists in grouping mesh vertices into spatial regions the segmentation of a mesh sequence can have various interpretations with respect to time and space. We propose here three different definitions. Let us first recall a formal definition of a static mesh segmentation.

Definition 1.2 (Segmentation of a static mesh [33]). Let $M=$ $(V, E, F)$ be a $3 D$ surface mesh. A segmentation $\Sigma$ of $M$ is the set of sub-meshes $\Sigma=\left\{M_{1}, \ldots, M_{k}\right\}$ induced by a partition of either $V$ or $E$ or $F$ into $k$ disjoint sub-sets. 

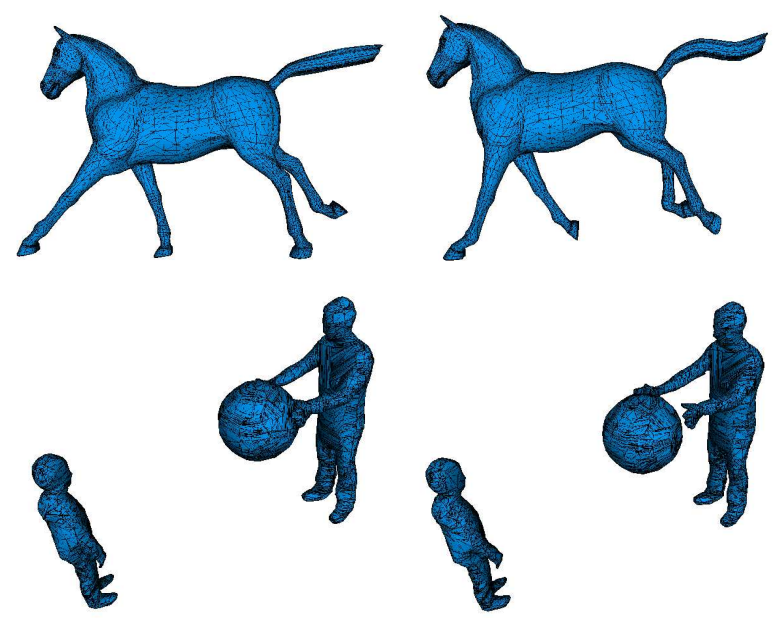

Figure 1: First row: two consecutive frames of a TCMS. Second row: two consecutive frames of a TIMS (in particular, notice the change in topology).

Definition 1.2 can be generalized in various ways to mesh sequences. For instance, the sequence itself can be partitionned into sub-sequences:

Definition 1.3 (Temporal segmentation). Let $M S=\left\{M^{i}, i=\right.$ $1 \ldots f\}$ be a mesh sequence. A temporal segmentation $\Sigma_{t}$ of $M S$ is a set of sub-sequences $\Sigma_{t}=\left\{M S_{1}, \ldots, M S_{k}\right\}$ such that $\forall j \in[1, k], M S_{j}=\left\{M^{i_{j}}, \ldots, M^{i_{j+1}-1}\right\}$ with $i_{1}=1<i_{2}<\cdots<$ $i_{k+1}=f+1$.

Possible applications of a temporal segmentation of a TIMS are mesh sequence decomposition into sub-sequences without topological changes or motion-based mesh sequence decomposition, as could be done for instance with the methods of Yamasaki and Aizawa [45] or Tung and Matsuyama [40].

In this paper, we are interested by geometric segmentations, that is to say the spatial segmentation of each mesh of the input sequence. We propose two different definitions.

Definition 1.4 (Coherent segmentation, variable segmentation). Let $M S=\left\{M^{i}, i=1 \ldots f\right\}$ be a mesh sequence. A coherent segmentation $\Sigma_{c}$ of $M S$ is a set of segmentations $\Sigma^{i}=$ $\left\{M_{1}^{i}, \ldots, M_{k_{i}}^{i}\right\}$ of each mesh $M^{i}$ of $M S$, such that:

- the number $k$ of sub-meshes is the same for all segmentations: $\forall i, j \in[1, f], k_{i}=k_{j}$;

- there is a one-to-one correspondence between sub-meshes of any two meshes;

- the connectivity of the segmentations, that is to say the neighborhood relationships between sub-meshes, is preserved over the sequence.

$A$ variable segmentation $\Sigma_{v}$ of $M S$ is a set of segmentations $\Sigma^{i}=$ $\left\{M_{1}^{i}, \ldots, M_{k_{i}}^{i}\right\}$ of each mesh $M^{i}$ of $M S$ which is not a coherent segmentation.

Note that our definition of a variable segmentation is very general. Intermediate mesh sequence segmentation definitions can be thought of, such as a sequence of successive coherent segmentations which would differ only for a few sub-meshes.

A coherent segmentation of a mesh sequence can be thought as a segmentation of some mesh of the sequence (for instance, the first one) which is mapped to the other meshes. Coherent segmentations are usually desired for shape analysis and understanding, when the overall structure of the shape is preserved during the deformation. However, variable segmentations can be helpful to display different information at each time step. For instance, they can be used to detect when changes in motion occur (see Figure $9(a, b, c)$ for an example), which is useful e.g. for animation compression or event detection with a CCTV system. In this paper, we propose a variable segmentation algorithm which recovers the decomposition of the motion over the sequence. For instance, two neighboring parts of the shape with different rigid motions are first put into different sub-meshes. They are later merged when they start sharing the same motion. Our algorithm can also create a coherent segmentation, which distinguishes between parts with different motion for at least a few meshes.

Please see the accompanying video for examples of coherent and variable segmentations.

\subsection{Contributions}

We propose an algorithm to compute a variable segmentation of a mesh sequence into components that move rigidly over time (section 3). This algorithm can also create a coherent segmentation of the mesh sequence. It applies to any types of mesh sequences though it was originally designed for the most general case of temporally incoherent mesh sequences, with possibly topology changes that occur over time. In contrast to existing approaches, it does not require any prior knowledge as input. Another contribution lies in the design of error metrics to assess the results of existing mesh sequence segmentation techniques (section 5).

\section{Related work}

Solutions have been proposed to decompose a static mesh into meaningful regions for motion (e.g., invariant under isometric deformations), e.g. [10, 3, 14, 17, 20, 31, 37, 12]. However and since our concern is the recovery of the rigid, or almost rigid, parts of a moving 3D shape, we focus in the following on approaches that consider deforming mesh sequence as input.

\subsection{Segmentation of temporally coherent mesh sequences}

Several methods have been proposed to compute motionbased coherent segmentation of temporally coherent mesh sequences. Among them, [23, 1, 19, 44, 32, 29] segment a TCMS into rigid components. In particular, de Aguiar [1] proposes a spectral approach which relies on the fact that the distance between two points is invariant under rigid transformation. In this paper, a spectral decomposition is also used (see Section 3.3.3). However, the invariant proposed by de Aguiar et al. cannot be used since mesh sequences without explicit temporal coherence are considered. 


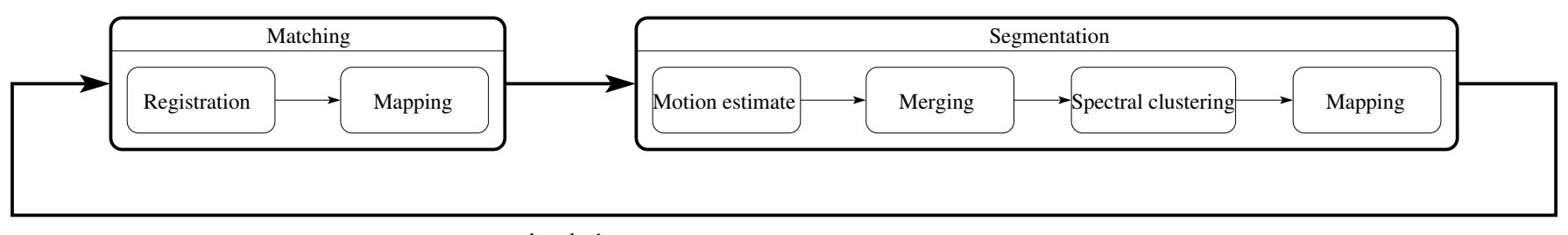

$\mathrm{k}:=\mathrm{k}+1$

Figure 2: Overall pipeline of our algorithm, at iteration $k, 1 \leq k<f$. As input we have meshes $M^{k}$, together with an initial segmentation estimate $\Sigma_{e s t}^{k}$, and $M^{k+1}$. As output we get a segmentation $\Sigma^{k}$ of $M^{k}$ and an initial segmentation estimate $\Sigma_{\text {est }}^{k+1}$ of $M^{k+1}$.

\subsection{Segmentation of temporally incoherent mesh sequences}

To solve the problem for temporally incoherent mesh sequences, a first strategy is to convert them to TCMS [43, 7]. While providing rich information for segmentation over time sequences, this usually requires a reference model that introduces an additional step in the acquisition pipeline, hence increasing the noise level. Moreover, the reference model usually strongly constrains shape evolution to a limited domain and does not allow for topology changes.

Only a few methods directly work on TIMS. Lee et al. [22] propose a segmentation method for TIMS using an additional skeleton as input. Franco and Boyer [13] propose to track and recover motion over a TIMS at the same time, hence creating a coherent segmentation, but the number of sub-meshes must be known. Varanasi and Boyer [42] segment a few meshes of a TIMS into convex parts, then register these regions to create a coherent segmentation. Their approach does not take into account the shape topology, thus the produced segmentation does not change with the topology. Tung and Matsuyama [41] handle topology changes, however their segmentation uses a learning step from training input sequences. In our work, we do not consider any a priori knowledge about the desired segmentation. In a previous work [2] we proposed a framework to segment a TIMS into rigid parts. As for the other works, our approach was only able to create coherent segmentations. In particular, it did not handle topology changes.

Another interesting work is Cuzzolin et al.'s method [11] that computes protrusion segmentation on point cloud sequences. This method is based on the detection of shape extremities, such as hands or legs. Our objective is different, it is to decompose it into rigidly moving parts.

\section{Mesh sequence segmentation}

In this section we describe our main contribution, that is a segmentation algorithm of a mesh sequence into rigidly moving components. Our algorithm takes as input a TIMS. This mesh sequence can include topology changes (genus and/or number of connected components of the meshes). It can produce either a variable or a coherent segmentation, depending on the user's choice.

\subsection{Overview}

We propose an iterative scheme that clusters vertices into rigid segments along a TIMS using motion information between successive meshes. For each mesh, rigid segments can be refined by separating parts that present inconsistent motions or otherwise merged when neighboring segments present similar motion. Motion information are estimated by matching meshes at successive instants. The main features of our algorithm are:

- it is fully automatic and does not require prior knowledge on the observed shape;

- it handles arbitrary shape evolutions, including changes in topology;

- it only requires a few meshes in memory at a time. Thus, segmentation can be computed on the fly and long sequences composed of meshes with a high number of vertices can be handled, see e.g. Figure 9 .

The algorithm alternates between two stages at iteration $k, 1 \leq k<f$ (see Figure 2, $f$ is the number of meshes in the sequence):

1. matching between 2 consecutive meshes $M^{k}$ and $M^{k+1}$ and computation of displacement vectors within a time window;

2. segmentation of $M^{k}$ and mapping to $M^{k+1}$.

Matching and segmentation algorithms are described in sections 3.2 and 3.3, respectively. This algorithm produces a variable segmentation. In case a coherent segmentation is needed, a post-processing stage is added (Section 3.4).

Four parameters can be tuned to drive the segmentation:

- the minimum segment size prevents the creation of too small segments. It is set to $4 \%$ of the total number of vertices of the current mesh in all our experiments. We noticed that this number is sufficient to avoid the creation of small segments around articulations, that are usually not rigid;

- the maximum subdivision of a segment prevents a segment to be split into too many small segments, when the motion becomes highly non rigid. It is set to 8 segments in all of our experiments;

- the eigengap value is used to determine the allowed motion variation within a segment. It thus affects the refinement of the segmentation (see Section 3.3.3 and Figures 10 and 12);

- the merge threshold is used to decide whether two segments represent the same motion and need to be merged (see Section 3.3.2). 
The notations used throughout the rest of the paper are the following:

- $f$ : the number of meshes in the sequence;

- $M^{k}$ : the $k^{\text {th }}$ mesh of the sequence (can be composed of several connected components);

- $M^{\prime k}$ : the $k^{\text {th }}$ mesh $M^{k}$ registered to $M^{k+1}$;

- $n v\left(M^{k}\right)$ : the number of vertices in $M^{k}$;

- $v_{i}^{(k)}:$ the vertex with index $i$ in $M^{k}$;

- $\operatorname{Ng}\left(v_{i}^{(k)}\right)$ : the 1-ring neighbors of vertex $v_{i}^{(k)}$.

Note that $k$ is always used as the index for a mesh, and $i$ and $j$ as the indices for vertices in a mesh.

\subsection{Mesh matching}

The objective of this stage is, given meshes $M^{k}$ and $M^{k+1}, k \in$ $[1, f-1]$, to provide a mapping from vertices $v_{i}^{(k)}$ to vertices $v_{j}^{(k+1)}$, and a possibly different mapping from vertices $v_{j}^{(k+1)}$ to vertices $v_{i}^{(k)}$. This mapping is further used to propagate segment labels over the sequence. We proceed iteratively according to the following successive steps (see Figure 3): first, meshes $M^{k}$ and $M^{k+1}$ are registered (vertices $v_{i}^{(k)}$ are moved to new locations $v_{i}^{\prime(k)}$ close to $\left.M^{k+1}\right)$, then displacement vectors and vertex correspondences are estimated. The following subsections detail these steps.

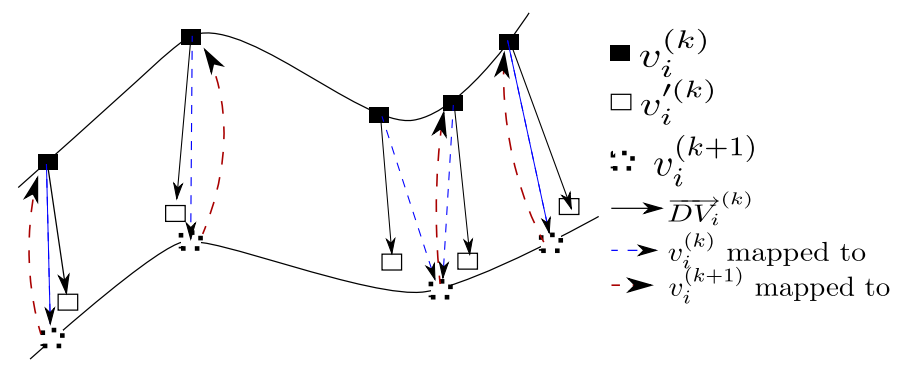

Figure 3: Matching process. Mesh $M^{k}$ with vertices $v_{i}^{(k)}$ is first registered to mesh $M^{k+1}$ with vertices $v_{i}^{(k+1)}$, inducing new vertices $v_{i}^{(k)}$. Displacement vectors $D V_{i}^{(k)}$ are defined thanks to this registration. Finally, mappings from $M^{k}$ to $M^{k+1}$ and from $M^{k+1}$ to $M^{k}$ are computed.

\subsubsection{Mesh registration}

The matching stage of our approach aims at establishing a dense cross parametrization between pairs of successive meshes of the sequence. Among the many available algorithms for this task, we chose to favor generality by casting the problem as the registration of two sets of points and normals. This means that we exclusively use geometric cues to align the two meshes, even when photometric information is available like in the case of meshes reconstructed from multi-camera systems. Thus, our approach also handles the case of software generated mesh sequences.

We implemented the method of Cagniart et al. [7] that iteratively deforms the mesh $M^{k}$ to fit the mesh $M^{k+1}$. This approach decouples the dimensionality of the deformation from the complexity of the input geometry by arbitrarily dividing the surface into elements called patches. Each of these patches is associated to a rigid frame that encodes for a local deformation with respect to the reference pose $M^{k}$. The optimization procedure is inspired by ICP as it iteratively re-estimates point correspondences between the deformed mesh and the target point set and then minimizes the distance between the two point sets while penalizing non rigid deformations of a patch with respect to its neighbors. Running this algorithm in a coarse-to-fine manner by varying the radii of the patches has proven in our experiments to robustly converge, and to be faster than using a single patch-subdivision level.

\subsubsection{Mappings and displacement vectors computation}

By using the previous stage, we get the registered mesh $M^{\prime k}$ of the mesh $M^{k}$ on mesh $M^{k+1}$. The displacement vector of each vertex $v_{i}^{(k)}$ in $M^{k}, 0 \leqslant i<n v\left(M^{k}\right)$ is then defined as:

$$
D V_{i}^{(k)}=v_{i}^{(k)}-v_{i}^{(k)}
$$

with $v_{i}^{(k)}$ the corresponding vertex in $M^{\prime k}$. To create a mapping from $M^{k}$ to $M^{k+1}$, the closest vertex in $M^{k+1}$ is found for each vertex $v_{i}^{\prime(k)}$ in $M^{\prime k}$ using Euclidean distance. A mapping from $M^{k+1}$ to $M^{k}$ is also created by finding for each vertex in $M^{k+1}$ the closest vertex in $M^{\prime k}$. Both mappings are necessary for the subsequent stage of our algorithm (see Sections 3.3.1 and 3.3.4). Note that mesh $M^{k+1}$ is not registered to mesh $M^{k}$ to compute the second mapping. Apart from saving computation time, this reduces inconsistencies between the two mappings: in most (though not all) cases, if $v_{i}^{(k)}$ is mapped to $v_{i}^{(k+1)}$, then $v_{i}^{(k+1)}$ is mapped to $v_{i}^{(k)}$. Also, note that these mappings are defined on the vertex sets. Hence, topology changes are not handled here. This is done in the next stage.

Using Euclidean distance instead of geodesic one may lead to occasional mismatchs. However, error hardly accumulates thanks to our handling of topology changes, see Section 3.3.4.

\subsection{Mesh segmentation}

In this part the goal is to create a segmentation $\Sigma^{k}$ of the mesh $M^{k}$ into rigidly moving components. The displacement vectors over a small time window computed during the previous stage are used, as well as (if $k \neq 1$ ) the segmentation $\Sigma^{k-1}$ of $M^{k-1}$ mapped to $M^{k}$ thanks to the bi-directional mapping between meshes $M^{k-1}$ and $M^{k}$. This provides an initial segmentation estimate $\Sigma_{e s t}^{k}$ of $M^{k}$. For $k=1$, the initial estimate is the trivial segmentation of $M^{1}$ into a single segment, containing all vertices $v_{i}^{(1)}$ of $M^{1}$.

We proceed in four successive steps. First, the motion of each vertex $v_{i}^{(k)}$ of $M^{k}$ is estimated using the displacements vectors (Section 3.3.1). Then, unless a coherent segmentation is required, neighboring segments in $\Sigma_{\text {est }}^{k}$ that present similar motions are merged (Section 3.3.2). Then a spectral clustering approach is used to refine the segmentation. This yields the segmentation $\Sigma^{k}$ of the vertices of $M^{k}$ (Section 3.3.3). Finally, $\Sigma^{k}$ is mapped onto $M^{k+1}$, to create the initial estimate $\Sigma_{e s t}^{k+1}$ of $\Sigma^{k+1}$ (Section 3.3.4). 
Our segmentation algorithm produces, by construction, connected segments since the atomic operations over segments are: merging neighboring segments (see Section 3.3.2) and splitting a segment into connected sub-segments (see Section 3.3.3).

\subsubsection{Motion estimate}

To estimate the motion of each vertex $v_{i}^{(k)}$ of $M^{k}$, the rigid transformation which maps $v_{i}^{(k)}$ together with its onering neighborhood $\mathrm{Ng}\left(v_{i}^{(k)}\right)$ onto $M^{\prime k}$ is computed, using Horn's method [16]. This method estimates a $4 \times 4$ matrix representing the best rigid transformation between 2 point clouds. A transformation matrix $T_{i}^{(k)}$ is therefore associated to each vertex $v_{i}^{(k)}$. With such a method however, computed estimates are noise sensitive, and slow motion is hardly detected. This is due to the fact that only the two meshes $M^{k}$ and $M^{\prime k}$ are used. In order to improve robustness of motion estimates, we propose to work on a time window. Motion is estimated from $M^{l}$ to $M^{\prime k}$, $M^{l}$ being the mesh where the segment has been created, either by splitting (see Section 3.3.3) or merging (see Section 3.3.2) of previous segments, or at the beginning of the process $(l=1)$. $l$ may be different for different vertices $v_{i}^{(k)}$ of $M^{k}$. Vertex $v_{j}^{(l)}$ of $M^{l}$ from which motion is estimated is defined using the previously computed bi-directional mapping. This method allows to detect slow motion (see Figure 4), and is less sensitive to noise and matching errors. Notice that different parts of the mesh may move with different speeds, this is not a problem as long as they belong to different segments, since the size of the time window is segment-dependent.
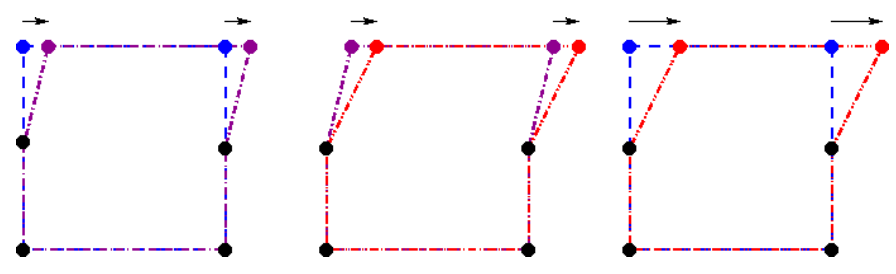

Figure 4: Three successive meshes $M^{k}$ (blue), $M^{k+1}$ (purple) and $M^{k+2}$ (red). Black dots correspond to vertices with null motion. Motion (black arrows) between $M^{k}$ and $M^{k+1}$, then between $M^{k+1}$ and $M^{k+2}$, is too slow to be detected by our subsequent stage (Section 3.3.3). Using a larger time window $[k, k+2]$ allows to detect this motion.

\subsubsection{Merging}

In the case of a variable segmentation, neighboring segments with similar motions before are merged at each time step refining the current segmentation. To this aim, the rigid transformation $T^{(k)}(S)$ of any segment $S$ is estimated over all its vertices, using Horn's method [16], as the rigid transformation $T_{i}^{(k)}$ of any vertex $v_{i}^{(k)}$ and its 1-ring neighborhood has been estimated. A greedy algorithm is then used:

- starting with the segment $S$ with the minimal residual error, this segment is merged with all neighboring segments $S^{\prime}$ such that $\left\|\log \left(T^{(k)}(S)^{-1} T^{(k)}\left(S^{\prime}\right)\right)\right\|<T_{\text {merge. }} T_{\text {merge }}$ is a user-defined threshold distance between the transformations of neighboring segments (see Section 3.1). The choice of this logarithm-based distance between transformations is explained in next section;

- the residual error for the new segment $S \cup \cup S^{\prime}$ is computed;

- we iterate, merging the next segment with the minimal residual error with its neighbors.

We stop when no merging is possible anymore. Note that this algorithm allows to handle topology changes such as merging of connected components.

The residual error for a segment $S$ corresponds to the mean distance, for all points $v_{i}^{(k)}$ of this segment, between the point $v_{i}^{(k+1)}$ and the location of $v_{i}^{(k)}$ after the computed rigid transformation $T^{(k)}(S)$ is applied:

$$
\operatorname{ResidualError}(S)=\frac{\sum_{v_{i}^{(k)} \in S}\left\|v_{i}^{(k+1)}-T^{(k)}(S) * v_{i}^{(k)}\right\|}{\operatorname{card}(S)}
$$

In our implementation, the choice of the threshold value $T_{\text {merge }}$ is left to the user. According to our experiments, it needs a few trials to find a suitable value. Choosing a high value merges most of the segments, while choosing a low value generates many clusters. The following values have been chosen for the displayed results in Sections 4 and 5: 0.03 for the $\mathrm{Bal}$ loon and the Horse sequences (Figures 9 and 11), 0.05 for the Dancer sequence (Figure 9) and 0.2 for the Cat sequence (Figure 13).

During the next step the current segmentation is refined. In order to prevent successive and useless merge and split of the same segments, we actually apply motion-based spectral clustering on detected pairs of segments to be merged before merging them. If the clustering results in some pairs splitting, then these pairs are not merged.

\subsubsection{Motion-based spectral clustering}

Spectral clustering is a popular and effective technique to robustly partition a graph according to some criterion [28]. It has been successfully applied to static meshes (see e.g. $[26,27,34,36])$, using the mesh vertices as the graph nodes and the mesh edges as the graph edges. The graph should be weighted with respect to the partition criterion. More precisely, edge weights represent similarity between their endpoints. In our case, these weights are related to the motion of neighboring vertices. This is in contrast to [1] where Euclidean distances between vertices are considered. In fact Euclidean distances can be preserved by non rigud transformation. Related to our approach is Brox and Malik's motion-based segmentation algorithm for videos [6].

Edge weights. To compute the weights $W^{(k)}$ of the graph edges, the following expression is used [30]:

$$
w_{i, j}^{(k)}= \begin{cases}\frac{1}{\left\|\log \left(T_{i}^{(k)-1} T_{j}^{(k)}\right)\right\|^{2}} & \text { if } i \neq j, \\ 0 & \text { if } i=j .\end{cases}
$$


As demonstrated in [30], this distance is mathematically founded since it corresponds to distances on the special $\mathrm{Eu}-$ clidean group of rigid transformations $S E(3)$.

Spectral clustering algorithm. Using the weighted adjacency matrix $W^{(k)}$, the normalized Laplacian matrix $L_{r w}^{(k)}$ is built as follows. Then the well-known Shi and Malik's normalized spectral clustering algorithm [35] is used to segment the graph.

$$
\begin{aligned}
& D_{i i}^{(k)}=\sum_{j \in \operatorname{Ng}\left(v_{i}^{(k)}\right)} w_{i j^{(k)}} . \\
& L^{(k)}=D^{(k)}-W^{(k)} . \\
& L_{r w}^{(k)}=D^{(k)-1} L^{(k)}=I^{(k)}-D^{(k)-1} W^{(k)} .
\end{aligned}
$$

Shi and Malik compute the first $K$ eigenvectors $u_{1}, \ldots, u_{K}$ of $L_{r w}^{(k)}$ and store them as columns of a matrix $U$. The rows $y_{i}, i=1 \ldots n$, of $U$ are then clustered using the classical $K$ means algorithm. Clusters for the input graph correspond to clusters of the rows $y_{i}$ : points $i$ such that $y_{i}$ belong to the same cluster are said to belong to the same segment of the graph.

This method assumes the number $K$ of clusters to be known. $K$ is computed using the classical eigengap method: let $\lambda_{1}, \lambda_{2}, \ldots, \lambda_{K}, \ldots$ be the eigenvalues of $L_{r w}^{(k)}$ ordered by increasing value, the smaller $K$ such that $\lambda_{K}-\lambda_{K-1}>$ eigengap is chosen. In our implementation, the eigengap value's choice is left to the user. In our experiments, a few trials (less than 5) were necessary to set this parameter. Two parameters are also used to prevent the creation of small segments in non-rigid areas (see Section 3.1): a minimum segment size and a maximum subdivision of a segment. According to our experiments, results are not very sensitive to the choice of these three parameters; the same values have been used for most of our experiments (see Section 4).

\subsubsection{Mapping to $M^{k+1}$}

The segmentation is computed at each time step on the current mesh $M^{k}$. Labels are then mapped onto the mesh $M^{k+1}$ using the bi-directional mapping defined in Section 3.2.2. Segments are first transferred using the mapping from $M^{k}$ to $M^{k+1}$. Then for all unmatched vertices in $M^{k+1}$, the mapping from $M^{k+1}$ to $M^{k}$ is used. Segments which are mapped on different connected components are split, see Figure 5. This allows us to naturally handle topology changes. This segmentation of $M^{k+1}$ serves as an initial estimate for the computation of $\Sigma^{k+1}$.

Note that segment splitting and merging allows to robustly handle mismatching, see Figure 6 . In case a vertex $v_{i}^{(k)}$ is wrongly matched to a vertex $v_{j}^{(k+1)}$, the corresponding segment is split in two. The new segment containing $v_{j}^{(k+1)}$ is then likely to be merged with a neighboring segment with similar motion.

\subsection{Coherent segmentation}

The algorithm can be modified to generate a coherent segmentation instead of a variable segmentation. This coherent segmentation clusters neighboring vertices that share similar rigid motion over the whole sequence. In other words, as long

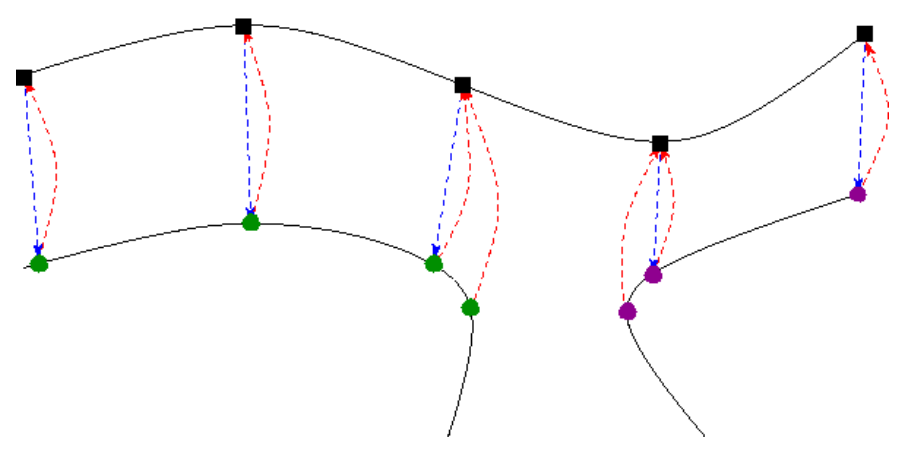

Figure 5: Splitting process. Blue and red arrows indicate the bi-directional mapping. The current segment (black squares) is split in two (green and magenta dots, respectively), since the three leftmost vertices and the two rightmost vertices are mapped to two different connected components.

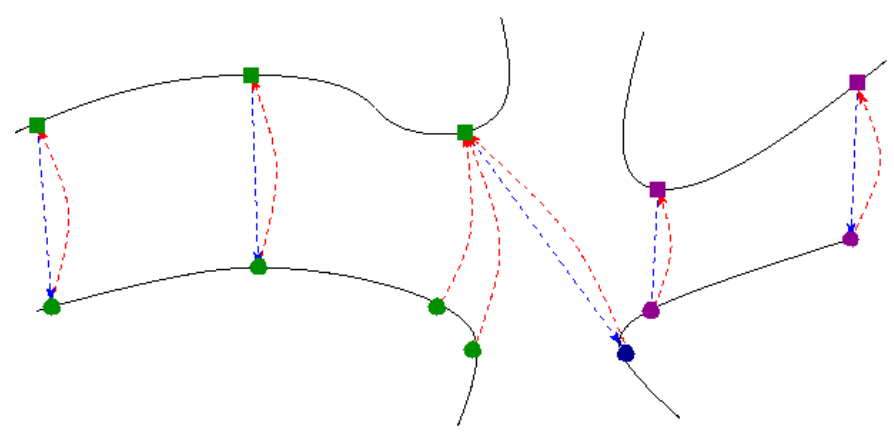

Figure 6: Segment splitting and merging allows to robustly handle mismatching. In case a vertex (rightmost green square) of $M^{k}$ is mismatched to a vertex (dark blue dot) of $M^{k+1}$, a new segment is created. This segment is then likely to be merged with the neighboring segment (magenta dots), since they present similar motions. 
as their motion differs over at least one small time window, two neighboring vertices do not belong to the same segment.

Creating a coherent segmentation is then straightforward. We only need:

- not to merge segments (step described in Section 3.3.2 is not applied);

- to map the segmentation $\Sigma^{f}$ of the last mesh $M^{f}$ back to the whole sequence.

To this purpose, the bi-directional mapping described in Section 3.2.2 is simply applied in reverse order, from $M^{f}$ to $M^{1}$. For each pair of successive meshes $\left(M^{k}, M^{k+1}\right)$ we first use the mapping from $M^{k+1}$ to $M^{k}$, then for all vertices of $M^{k}$ which are not assigned to a segment, the mapping from $M^{k}$ to $M^{k+1}$ is used.

\section{Results}

In this section we show and discuss visual results of our algorithm. A quantitative evaluation of these results is discussed in the next section. We first examine matching results, then segmentation results on difficult cases (temporally incoherent mesh sequences with topological changes, acquired from real data). We also show that our results on temporally coherent mesh sequences are visually similar to state-of-the-art approaches.

\subsection{Matching results}

The vertex matching computation is an important step since our segmentation algorithm relies on it (see Figure 2). Figure 7 shows the result of vertex matching between two successive meshes of a TIMS. Computation time is about 30 seconds for two meshes with approximately 7000 vertices each. This outperforms the matching method proposed in [2] which takes about 13 minutes to complete computation with the same data, for a similar result. Note that outliers in the matching are not explicitly taken into account in the segmentation, however their influence is limited by the threshold on the minimum segment size (see Section 3.1) that tends to force them to merge with neighboring segments.

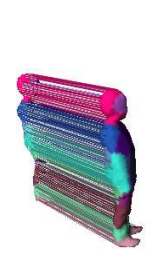

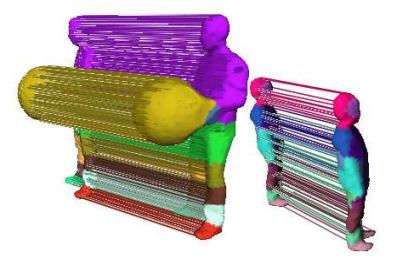

(a)

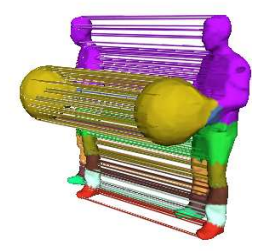

(b)
Figure 7: Result of vertex matching on real data captured from video cameras. (a) Full display. (b) Partial display.

Figure 8 shows a matching result between two consecutive frames of a sequence where the vertex density differs drastically. Even if vertex-to-vertex matching is less accurate than vertex-to-face matching (that is to say, matching every vertex of $M^{k}$ to the closest point of $M^{k+1}$, which can lie on an edge or inside a face), in our experiments it has proved to be sufficient for our purpose. Meanwhile, its computation is much faster.

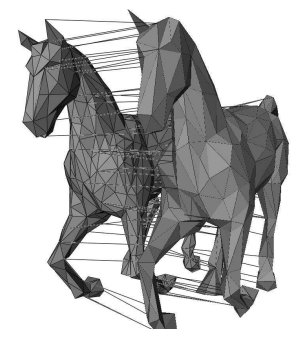

Figure 8: Result of vertex matching between two consecutive frames of a sequence with varying vertex density.

\subsection{Segmentations of TIMS with topology changes}

Figure 9 shows variable segmentations computed on two Balloon and Dancer sequences. Figure 10 shows coherent segmentations computed on the Balloon sequence. By construction, coherent segmentations contain more segments than variable segmentations since no merging operation occurs. Parameters for both variable and coherent segmentations of the Balloon sequence have the same values, except for the eigengap threshold that is slightly lower in the variable segmentation case $(0.40$ vs. 0.48 for result shown on Figure 10 (a)). According to our experiments, suitable parameter values for a given sequence are found in a few trials. The computation time of one mesh segmentation of the Dancer sequence is approximately 3 minutes with a (not optimized) Matlab implementation. Additional results appear in the accompanying video. Our algorithm does not require the whole sequence in memory at a given time step $k$, but only previous meshes which share at least one segment with the current segmentation, in addition to the next mesh (namely, meshes from $M^{l}$ to $M^{k+1}$, see Section 3.3.1). Thus, it can handle long sequences with a high number of vertices, such as the Balloon sequence which contains 300 meshes with approximately 15,000 vertices each.

Timings are given in the following table. The algorithm was implemented using Matlab on a laptop with a one-core 2.13 $\mathrm{GHz}$ processor.

\begin{tabular}{|l|c|}
\hline Segmentation & Total computation time \\
\hline Fig. $9(\mathrm{a}-\mathrm{c})$ & $43 \min 14$ \\
\hline Fig. $9(\mathrm{~d}-\mathrm{g})$ & $76 \min 48$ \\
\hline Fig. $12(\mathrm{a})$ & $29 \min 07$ \\
\hline Fig. $12(\mathrm{~b})$ & $25 \min 57$ \\
\hline Fig. 13 (a) & $3 \min 46$ \\
\hline
\end{tabular}

\subsection{Segmentation of TCMS}

Although our approach is designed for general cases, it can also handle TCMS and obtains visually similar results to previous TCMS-dedicated methods, as shown in Figure 11.

Figure 12 illustrates the influence of the eigengap threshold: the higher the eigengap value, the coarser the segmentation. 


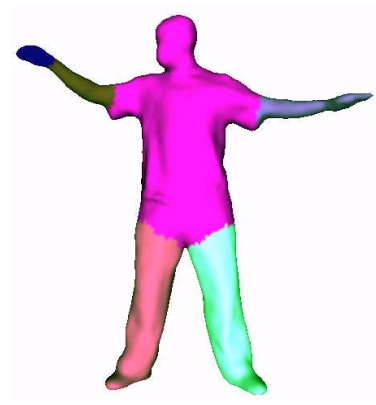

(a)

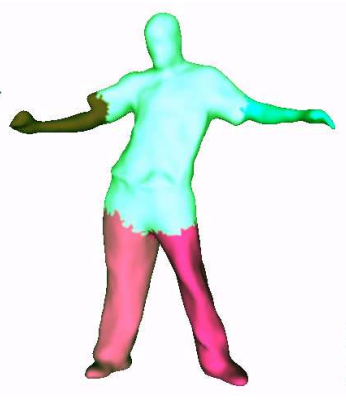

(b)

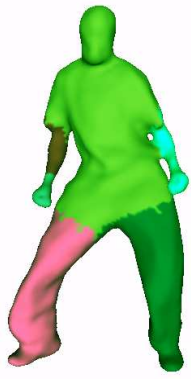

(c)
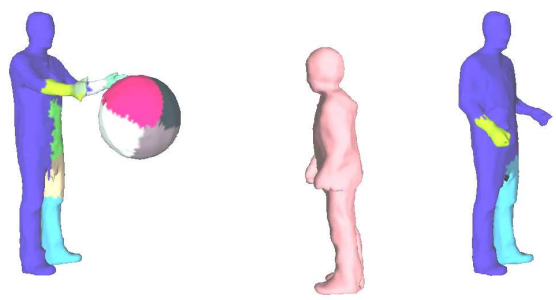

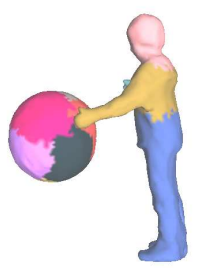

(e)

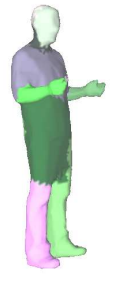

(f)

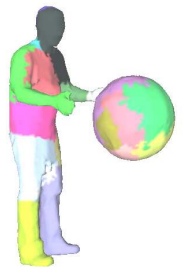

$(\mathrm{g})$

Figure 9: (a,b,c) Variable segmentation generated by our algorithm on the Dancer sequence [38]. First meshes are decomposed into 6 segments, then the right arm and right hand segments merge since they move the same way. Finally, this segment is split again. Note that topology changes can be handled (in the last meshes, the left arm is connected to the body). (d,e,f,g): Variable segmentation of a sequence with 15,000 vertices per mesh and topology changes. The balloon is over-segmented because its motion is highly non rigid.
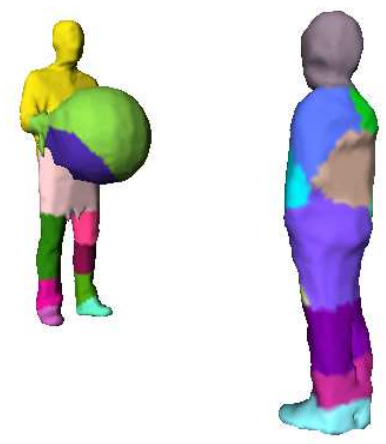

(a)

(b)

Figure 10: Coherent segmentation results on the Balloon sequence, obtained with two different eigengap values: (a) 0.48 , (b) 0.8 . Segments cluster neighboring vertices that share the same motion over the whole sequence.

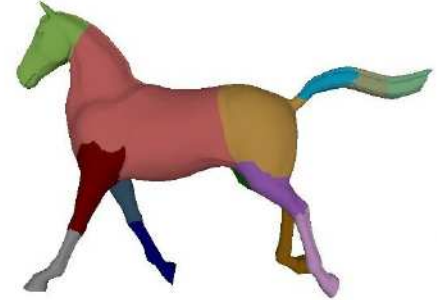

(a)

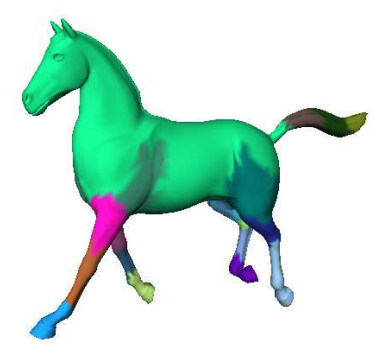

(c)

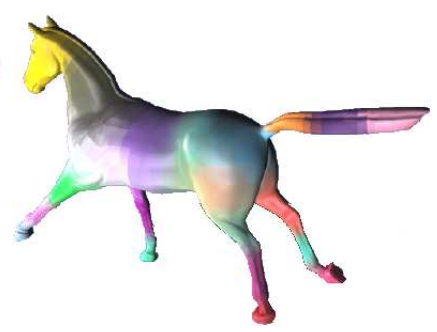

(b)

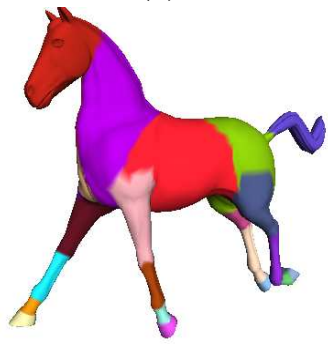

(d)
Figure 11: Segmentation results on a TCMS. (a) [23]. (b) [1]. (c) [2]. (d) Our method. 


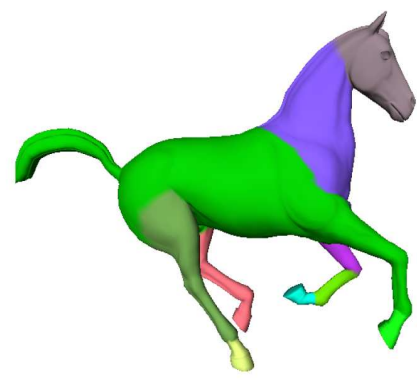

(a)

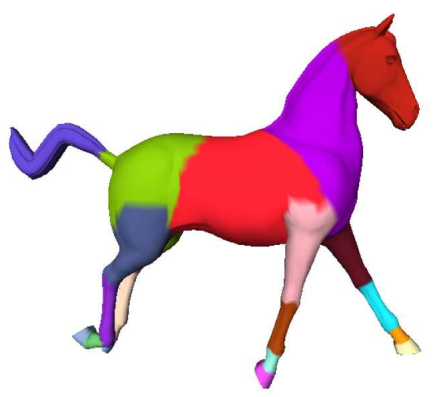

(b)
Figure 12: Segmentation of the Horse sequence [39] with two different eigengap values. (a) eigengap $=0.7$. (b) eigengap $=0.5$.

\section{Evaluation}

A quantitative and objective comparison of segmentation methods is an ill-posed problem since there is no common definition of what an optimal segmentation should be in the general case. Segmentation evaluation has been recently addressed in the static case using ground truth (i.e. segmentations defined by humans) [5, 9]. In the mesh sequence case, none of the previously cited articles in Section 2 proposes an evaluation of the obtained segmentations. We thus propose the following framework to evaluate a mesh sequence segmentation method.

\subsection{Optimal segmentation}

The optimal segmentation of a mesh sequence, be it a TCMS or a TIMS, into rigid components can be guessed when the motion and/or the kinematic structure is known. This is, for instance, the case with skeleton-based mesh animations, as created in the computer graphics industry. In this case, each mesh vertex of the sequence is attached to at least one (usually, no more than 4) joints of the animation skeleton, with given weights called skinning weights. These joints are organized in a hierarchy, which is represented by the "bones" of the skeleton that are, therefore, directed. For our evaluation, we attach each vertex to only one joint among the related joints, the furthest in the hierarchy from the root joint. If this joint is not unique, the one with the greatest skinning weight is kept. Each joint has its own motion, but several joints can move together in a rigid manner. For a given mesh, cluster joints of the animation skeleton can therefore be clustered into joint sets, each joint set representing a different motion. We now define as an optimal segment the set of vertices related to joints in the same joint set. Since the motion of each joint is known, we exactly know, for each mesh of the animation, what are the optimal segments.

This definition can be applied in the general case of TIMS, provided that each vertex of each mesh can be attached to a joint. However, we only tested it in the more convenient case of a TCMS.

\subsection{Error metrics}

We propose the following three metrics in order to evaluate a given segmentation with respect to the previously defined optimal segmentation:
- Assignment Error (AE): for a given mesh, the ratio of vertices which are not assigned to the correct segment. This includes the case of segments which are not created, or which are wrongly created;

- Global Assignment Error (GAE): the mean AE among all meshes of the sequence;

- Vertex Assignment Confidence (VAC): for a given vertex of a TCMS, the ratio of meshes in which the vertex is assigned to the correct segment.

$\mathrm{AE}$ and GAE give a quantitative evaluation of a mesh segmentation and the mesh sequence segmentation, respectively, with respect to the optimal segmentation. VAC can help to locate wrongly segmented areas.

Note that more sophisticated evaluation metrics exist to compare two static mesh segmentations [9]. We define AE as a simple ratio for sake of simplicity, but other metrics can also be used to define global assignment errors.

\subsection{Evaluation results}

We tested our algorithm on a walking cat skeleton-based animation (see Figure 13 and the accompanying video). We get a variable segmentation with a $\mathrm{AE}$ up to $17 \%$, in the worst case. Wrongly assigned vertices correspond to the cat skin around joints and to a wrong subdivision in cat paw, i.e. in the less rigid areas.

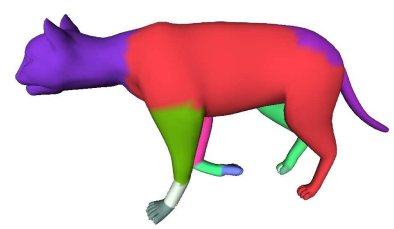

(a)

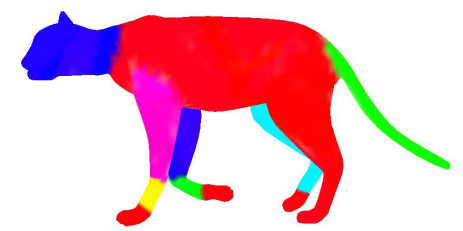

(b)
Figure 13: Result on a skeleton-based synthetic animation. (a) Computed variable segmentation. (b) Optimal variable segmentation, for the same mesh of the sequence.

In the case of coherent segmentations, and if matching issues are not taken into account, then the AE is the same for all meshes. Therefore, the GAE is equal to the AE of any mesh. For the cat sequence, the GAE is also $17 \%$. The VAC can be $0 \%$ or $100 \%$, and is only relevant as a relative criterion to compare vertices and find ill-segmented areas. On the cat sequence vertices in rigid areas (paws, tail, body) are often always assigned to the correct segment; their confidence is equal to 1 . In contrast, some vertices around joints can be assigned to the same neighboring segment in all meshes; their confidence drops to 0 , see Figure 14. We also computed these metrics for the method described in [2], using the same cat sequence. The GAE reaches $42 \%$, while the VAC can also be $0 \%$ or $100 \%$.

\section{Conclusion}

In this paper we addressed the problem of $3 \mathrm{D}$ mesh sequence segmentation into rigidly moving components. We have proposed a classification of mesh sequence segmentations, together 


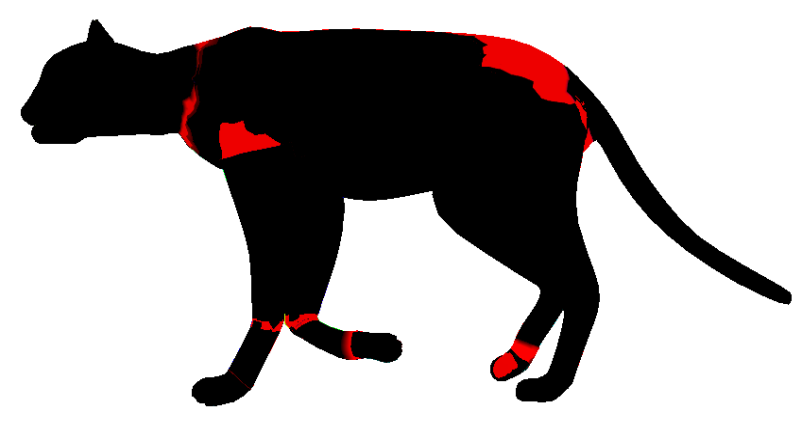

Figure 14: Vertex Assignment Confidence results. Vertices for which VAC is 0 are colored in red, while vertices with confidence equal to 1 are in black.

with a segmentation method that takes as input a mesh sequence, even when no explicit temporal coherence is available, and possibly with topology changes. This method produces either a coherent or a variable segmentation into rigid components depending on the user's choice. It uses a few parameters which can be set in a few trials, according to our experiments. We have also proposed a framework for quantitative evaluations of rigid segmentation methods.

\subsection{Current limitations}

We are currently aware of three limitations in the proposed algorithm:

- our method clearly depends on the quality of the matching process. Important errors in matching computation may lead to wrong results;

- segmentation can slightly drift: this is due to the fact that only 2 meshes are considered when matching;

- segments which are wrongly subdivided are transferred to the following meshes, meaning that errors on an early mesh in the sequence can affect the whole segmentation. Such errors are generally due to errors in the matching process. This issue is less critical on variable segmentations than on coherent segmentations, since segments are merged later.

Figure 15 shows an example of these limitations. In this example, the entire left front leg of the horse at frame $k$ was intentionally mismatched to the right front leg at frame $k+1$, and vice-versa. Resulting erroneous segmentation at frame $k+1$ is then propagated to the following frames, since no merging with the neighboring segment occurs. Fortunately, this problem seldom happens. As shown in our quantitative evaluations, using the matching process described in Section 3.2, vertices that are wrongly assigned to a segment are located near articulations. Vertices in rigid regions are generally correctly clustered.

Despite these limitations, our method has shown as good results as current state-of-the-art methods on temporally coherent mesh sequences (see Figure 11), although it has been designed for the more difficult case of mesh sequences without temporal coherence.

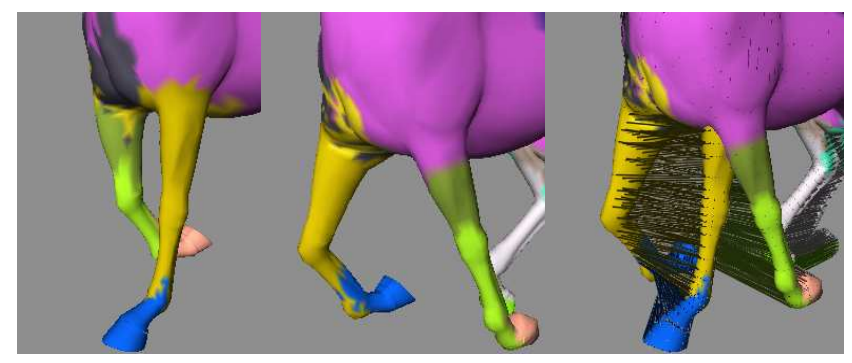

(a)

(b)

(c)

Figure 15: Matching error and resulting coherent segmentation. (a,b) Two consecutive frames of the Horse sequence. (c) Matching between these two frames.

\subsection{Future work}

Our method can be improved in various ways. As explained above, it would be interesting to improve the vertex assignments around articulations. Adding prior knowledge about the geometry of desired segments (e.g. cylindrical shape, or symmetry information) would be helpful to enhance the robustness of the method. It would also be useful to reduce the number of parameters. Our algorithm handles topology changes, but our solution is not semantically satisfactory in case a new connected component (e.g., the shade of the balloon in the Balloon sequence) appears, since it is first attached to an existing segment before being split from it.

We hope our evaluation metrics would be helpful for further work in the domain. However, a more in-depth study of the three proposed criteria need to be performed to assess their usefulness. Finally, a user validation can also help to quantify segmentations produced by our algorithm.

\section{Acknowledgments}

The Balloon sequence is courtesy of Inria Grenoble [18]. The Dancer sequence is courtesy of University of Surrey [38]. The Horse sequence is courtesy of M.I.T. [39]. The Cat sequence is courtesy of Inria Grenoble [4]. This work has been partially funded by the french National Reseach Agency (ANR) through the MADRAS (ANR-07-MDCO-015) and MORPHO (ANR10-BLAN-0206) projects.

\section{References}

[1] de Aguiar, E., Theobalt, C., Thrun, S., Seidel, H., 2008. Automatic conversion of mesh animations into skeleton-based animations. Computer Graphics Forum (Eurographics proceedings) 27.

[2] Arcila, R., Buddha, K., Hétroy, F., Denis, F., Dupont, F., 2010. A framework for motion-based mesh sequence segmentation, in: Proceedings of the International Conference on Computer Graphics, Visualization and Computer Vision (WSCG).

[3] Attene, M., Falcidieno, B., Spagnuolo, M., 2006. Hierarchical mesh segmentation based on fitting primitives. The Visual Computer 22.

[4] Aujay, G., Hétroy, F., Lazarus, F., Depraz, C., 2007. Harmonic skeleton for realistic character animation, in: Proceedings of the Symposium on Computer Animation (SCA).

[5] Benhabiles, H., Vandeborre, J., Lavoué, G., Daoudi, M., 2009. A framework for the objective evaluation of segmentation algorithms using a ground-truth of human segmented 3D-models, in: Proceedings of the IEEE International Conference on Shape Modeling and Applications (SMI). 
[6] Brox, T., Malik, J., 2010. Object segmentation by long term analysis of point trajectories, in: Proceedings of the European Conference on Computer Vision (ECCV)

[7] Cagniart, C., Boyer, E., Ilic, S., 2010. Iterative deformable surface tracking in multi-view setups, in: Proceedings of the International Symposium on 3D Data Processing, Visualization and Transmission (3DPVT).

[8] Cashman, T., Hormann, K., 2012. A continuous, editable representation for deforming mesh sequences with separate signals for time, pose and shape. Computer Graphics Forum (Eurographics proceedings) 31.

[9] Chen, X., Golovinskiy, A., Funkhouser, T., 2009. A benchmark for 3d mesh segmentation. ACM Transactions on Graphics (SIGGRAPH proceedings) 28 .

[10] Cutzu, F., 2000. Computing 3d object parts from similarities among object views, in: Proceedings of the IEEE Conference on Computer Vision and Pattern Recognition (CVPR).

[11] Cuzzolin, F., Mateus, D., Knossow, D., Boyer, E., Horaud, R., 2008. Coherent laplacian 3-d protrusion segmentation, in: Proceedings of the IEEE Conference on Computer Vision and Pattern Recognition (CVPR).

[12] Fang, Y., Sun, M., Kim, M., Ramani, K., 2011. Heat mapping: a robust approach toward perceptually consistent mesh segmentation, in: Proceedings of the IEEE Conference on Computer Vision and Pattern Recognition (CVPR).

[13] Franco, J., Boyer, E., 2011. Learning temporally consistent rigidities, in: Proceedings of the IEEE Conference on Computer Vision and Pattern Recognition (CVPR).

[14] de Goes, F., Goldenstein, S., Velho, L., 2008. A hierarchical segmentation of articulated bodies. Computer Graphics Forum (Symposium on Geometry Processing proceedings) 27.

[15] Goldenthal, R., Harmon, D., Fattal, R., Bercovier, M., Grinspun, E., 2007. Efficient simulation of inextensible cloth. ACM Transactions on Graphics (SIGGRAPH proceedings) 26.

[16] Horn, B., 1987. Closed-form solution of absolute orientation using unit quaternions. J. Opt. Soc. Am. A 4

[17] Huang, Q., Wicke, M., Adams, B., Guibas, L., 2009. Shape decomposition using modal analysis. Computer Graphics Forum (Eurographics proceedings) 28 .

[18] Inria, . Balloon sequence. http://4drepository.inrialpes.fr/.

[19] Kalafatlar, E., Yemez, Y., 2010. 3d articulated shape segmentation using motion information, in: Proceedings of the International Conference on Pattern Recognition (ICPR).

[20] Kalogerakis, E., Hertzmann, A., Singh, K., 2010. Learning 3d mesh segmentation and labeling. ACM Transactions on Graphics (SIGGRAPH proceedings) 29.

[21] Kircher, S., Garland, M., 2006. Editing arbitrarily deforming surface animations. ACM Transactions on Graphics (SIGGRAPH proceedings) 25 .

[22] Lee, N., T.Yamasaki, Aizawa, K., 2008. Hierarchical mesh decomposition and motion tracking for time-varying-meshes, in: Proceedings of the IEEE International Conference on Multimedia and Expo (ICME).

[23] Lee, T.Y., Wang, Y.S., Chen, T.G., 2006. Segmenting a deforming mesh into near-rigid components. The Visual Computer 22.

[24] Lengyel, J., 1999. Compression of time-dependent geometry, in: Proceedings of the Symposium on Interactive 3D graphics (I3D).

[25] Lewis, J., Cordner, M., Fong, N., 2000. Pose-space deformation: a unified approach to shape interpolation and skeleton-driven deformation, in: Proceedings of SIGGRAPH.

[26] Liu, R., Zhang, H., 2004. Segmentation of 3d meshes through spectral clustering, in: Proceedings of Pacific Graphics.

[27] Liu, R., Zhang, H., 2007. Mesh segmentation via spectral embedding and contour analysis. Computer Graphics Forum (Eurographics proceedings) 26.

[28] von Luxburg, U., 2007. A tutorial on spectral clustering. Statistics and Computing 17.

[29] Marras, S., Bronstein, M.M., Hormann, K., Scateni, R., Scopigno, R., 2012. Motion-based mesh segmentation using augmented silhouettes. Graphical Models .

[30] Murray, R., Sastry, S., Zexiang, L., 1994. A Mathematical Introduction to Robotic Manipulation. CRC Press, Inc.

[31] Reuter, M., 2010. Hierarchical shape segmentation and registration via topological features of laplace-beltrami eigenfunctions. International Journal of Computer Vision 89.
[32] Rosman, G., Bronstein, M.M., Bronstein, A.M., Wolf, A., Kimmel, R., 2011. Group-valued regularization framework for motion segmentation of dynamic non-rigid shapes, in: Scale Space and Variational Methods in Computer Vision.

[33] Shamir, A., 2008. A survey on mesh segmentation techniques. Computer Graphics Forum 27.

[34] Sharma, A., von Lavante, E., Horaud, R., 2010. Learning shape segmentation using constrained spectral clustering and probabilistic label transfer, in: Proceedings of the European Conference on Computer Vision (ECCV).

[35] Shi, J., Malik, J., 2000. Normalized cuts and image segmentation. IEEE Transactions on Pattern Analysis and Machine Intelligence (PAMI) 22.

[36] Sidi, O., van Kaick, O., Kleiman, Y., Zhang, H., Cohen-Or, D., 2011. Unsupervised co-segmentation of a set of shapes via descriptor-space spectral clustering. ACM Transactions on Graphics (SIGGRAPH Asia proceedings) 30 .

[37] Skraba, P., Ovsjanikov, M., Chazal, F., Guibas, L., 2010. Persistencebased segmentation of deformable shapes, in: CVPR Workshop on NonRigid Shape Analysis and Deformable Image Alignment.

[38] Starck, J., Hilton, A., 2007. Surface capture for performance-based animation. IEEE Computer Graphics and Applications .

[39] Sumner, R., Popović, J., 2004. Deformation transfer for triangle meshes. ACM Transactions on Graphics (SIGGRAPH proceedings) 23.

[40] Tung, T., Matsuyama, T., 2009. Topology dictionary with markov model for $3 \mathrm{~d}$ video content-based skimming and description, in: Proceedings of the IEEE Conference on Computer Vision and Pattern Recognition (CVPR).

[41] Tung, T., Matsuyama, T., 2010. 3d video performance segmentation, in: Proceedings of the IEEE International Conference on Image Processing (ICIP).

[42] Varanasi, K., Boyer, E., 2010. Temporally coherent segmentation of 3d reconstructions, in: Proceedings of the International Symposium on 3D Data Processing, Visualization and Transmission (3DPVT).

[43] Vlasic, D., Baran, I., Matusik, W., Popović, J., 2008. Articulated mesh animation from multi-view silhouettes. ACM Transactions on Graphics (SIGGRAPH proceedings) 27.

[44] Wuhrer, S., Brunton, A., 2010. Segmenting animated objects into nearrigid components. The Visual Computer .

[45] Yamasaki, T., Aizawa, K., 2007. Motion segmentation and retrieval for $3 \mathrm{~d}$ video based on modified shape distribution. EURASIP Journal on Applied Signal Processing . 